\title{
Bidirectional Synthesis of the IJK Fragment of Ciguatoxin CTX3C by Sequential Double Ring-Closing Metathesis and Tsuji-Trost Allylation
}

\author{
Michael Popadynec, Helen Gibbard, and J. Stephen Clark*
}

Cite This: Org. Lett. 2020, 22, 3734-3738

Read Online

ABSTRACT: A novel four-step bidirectional strategy has been used to synthesize the IJK fragment of the marine polyether natural product CTX3C from a simple monocyclic precursor in a concise and efficient manner. The four-step bidirectional sequence involves ring-closing metathesis, alcohol oxidation, enol carbonate formation, and palladium-mediated Tsuji-Trost allylation.

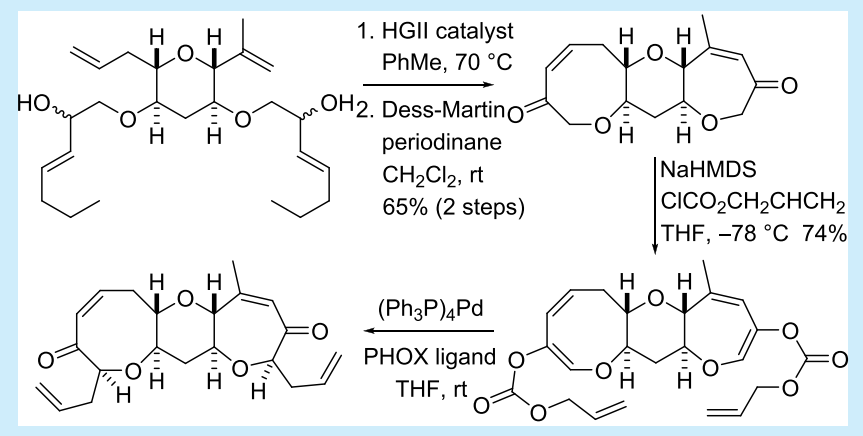

$\mathrm{T}$ he fused polyether natural product CTX3C is one of more than a dozen ciguatoxins to have been isolated from algae or fish collected in tropical or subtropical regions of the Pacific Ocean or Indian Ocean, or from samples collected in the Caribbean Sea (Figure 1). ${ }^{1,2}$ The ciguatoxins are extremely potent neurotoxins, and in common with many other large fused polyether natural products of marine origin (e.g., the brevetoxins), they disrupt the transmission of nerve signals by binding at site 5 on voltage-sensitive sodium channels. ${ }^{3}$ Human exposure to the ciguatoxins by ingestion of contaminated fish or seafood results in a condition known as ciguatera in which sufferers exhibit severe neurological, gastrointestinal, and cardiovascular problems; fatalities, although rare, have been recorded. ${ }^{4}$

CTX3C was first isolated from a culture of the marine dinoflagellate Gambierdiscus toxicus and characterized by Yasumoto and co-workers in 1993 (Figure 1). ${ }^{1}$ The molecular structure of CTX3C comprises 13 fused cyclic ethers, with ring sizes that vary from five to nine, and is typical of the ciguatoxin family. The size, complexity, and number of medium-sized cyclic ethers embedded in its structure make CTX3C an extremely challenging yet alluring target for total synthesis. Although several groups have synthesized major portions of the natural product over the past two decades, ${ }^{5}$ the crowning achievements in this area are undoubtedly the impressive and elegant total syntheses of CTX3C and 51-hydroxy-CTX3C, completed by Hirama and co-workers, ${ }^{6}$ and the more recent total synthesis of CXT1B, completed by Isobe and Hamajima.?

As part of our general program concerning the development of new reactions and strategies for the rapid and efficient synthesis of fused polycyclic ether arrays, we have explored the use of iterative and bidirectional approaches to synthesize large fragments of the gambieric acids and CTX3C., ${ }^{8,9}$ In our previous work concerning the synthesis of CTX3C, the $\mathrm{A}-\mathrm{E}$ fragment of CTX3C was assembled by a route in which ringclosing metathesis (RCM) reactions were used to construct four of the five rings including the medium-sized A, D, and E rings. ${ }^{9}$ We now present a novel approach to the synthesis of the IJK fragment of CTX3C in which a four-step sequence is performed bidirectionally to assemble this key tricyclic fragment from a simple monocyclic precursor.

Our retrosynthetic analysis of CTX3C is shown in Figure 1. Disconnection through the rings $\mathrm{G}$ and $\mathrm{H}$ implies that the fused 13-ring framework will be constructed by coupling of an A-F fragment $i$ with an $\mathrm{I}-\mathrm{M}$ fragment ii followed by formation of rings $\mathrm{G}$ and $\mathrm{H}$. Further disconnection of the I-M fragment ii through the LM spiroacetal then reveals the tricyclic fragment iii and removal of the methyl substituent from ring I, and the hydroxyl group from ring $\mathrm{K}$ suggests bisenone iv as a key intermediate. Removal of the side chains and scission of both enones then reveals the J-ring fragment $\mathbf{v}$.

Implicit in our retrosynthetic analysis of the I-M fragment is formation of rings I and K by RCM and installation of the side chains of both rings by Tsuji-Trost allylation, ${ }^{10}$ a reaction that we have already demonstrated as being highly effective for the

Received: April 7, 2020

Published: April 19, 2020 


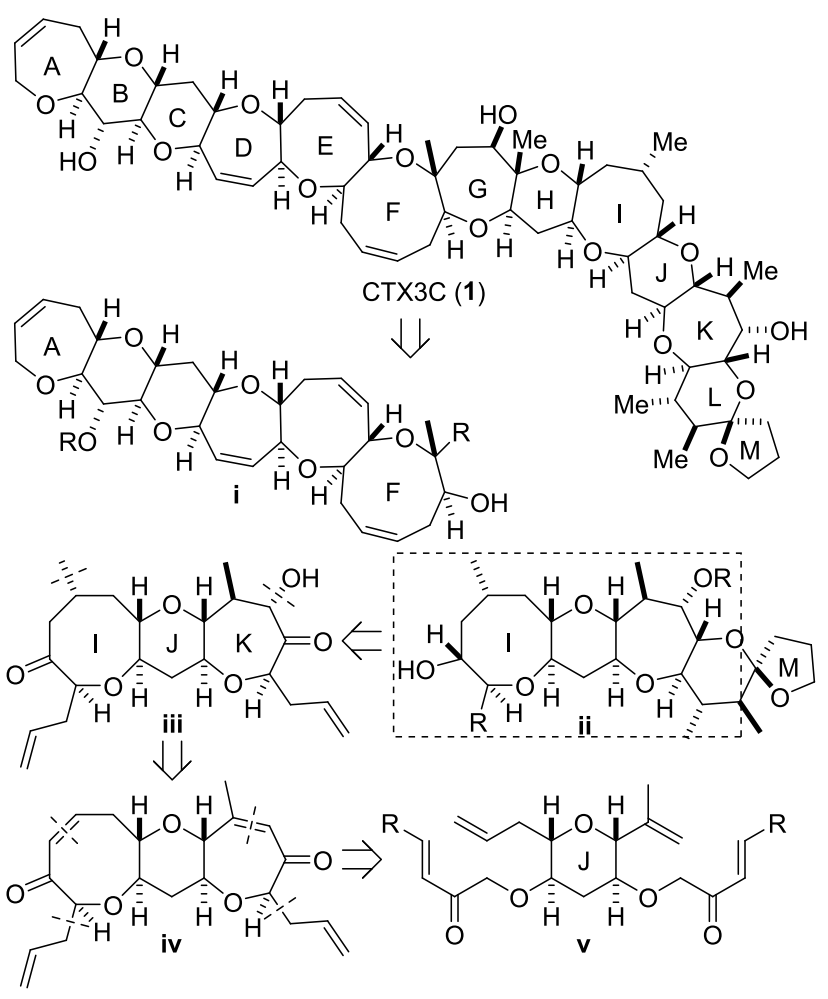

Figure 1. Disconnection of ciguatoxin CTX-3C (1) to reveal the IJK fragment iv and further disconnection to I-ring fragment $\mathbf{v}$.

stereoselective functionalization of seven- and eight-membered cyclic ethers. ${ }^{11}$ We chose to pursue a highly ambitious approach in which RCM, Tsuji-Trost allylation, and the intermediate reactions would be performed bidirectionally in order to minimize the number of steps.

The synthesis of the precursor required for the bidirectional sequence commenced with the enantiomerically pure enol ether 1, which was prepared in two steps from commercially available tri-O-acetyl-D-glucal (Scheme 1). ${ }^{8 \mathrm{~b}}$ The 1,3-diol was converted into an acetonide and the enol ether was epoxidized thereafter by treatment with $m$-CPBA in the presence of $\mathrm{KF}$ according to a procedure used by Chiappe and co-workers for the epoxidation of structurally related glycals. ${ }^{12}$ This reaction was chosen because it was more amenable to scale-up than the more widely used DMDO epoxidation procedure, and the unstable epoxides could be isolated more rapidly. ${ }^{13}$ The resulting mixture of diastereomeric glycal epoxides $\mathbf{2 a}$ and $\mathbf{2 b}$ was treated directly with allylmagnesium chloride to produce the diastereomeric alcohols $\mathbf{3 a}$ and $\mathbf{3 b}$ in a combined yield of $75 \%$ over two steps. The alcohol $3 \mathbf{b}$ was recovered and converted into the required diastereomer $3 \mathbf{a}$ by sequential oxidation, epimerization, and reduction. The alcohol $3 \mathbf{3 a}$ was then converted into the corresponding triol by acid-mediated cleavage of the acetonide. Persilylation was performed by treatment of the triol with 4 equiv of tert-butylydimethylsilyl chloride, an excess of imidazole, and a substoichiometric amount of DMAP to deliver the tetrahydropyran 4. Selective monodeprotection to give the primary alcohol 5 was accomplished by treatment of the intermediate 4 with camphorsulfonic acid. ${ }^{14}$

The precursors $(11 \mathrm{a}-\mathrm{c}$ and 12$)$ required for exploration of the bidirectional $\mathrm{RCM}$ reaction were prepared from the alcohol $\mathbf{5}$ as shown in Scheme 2. The primary alcohol $\mathbf{5}$ was converted into a diastereomeric mixture of the secondary
Scheme 1. Synthesis of the Alcohol 5a from the Glucal 1

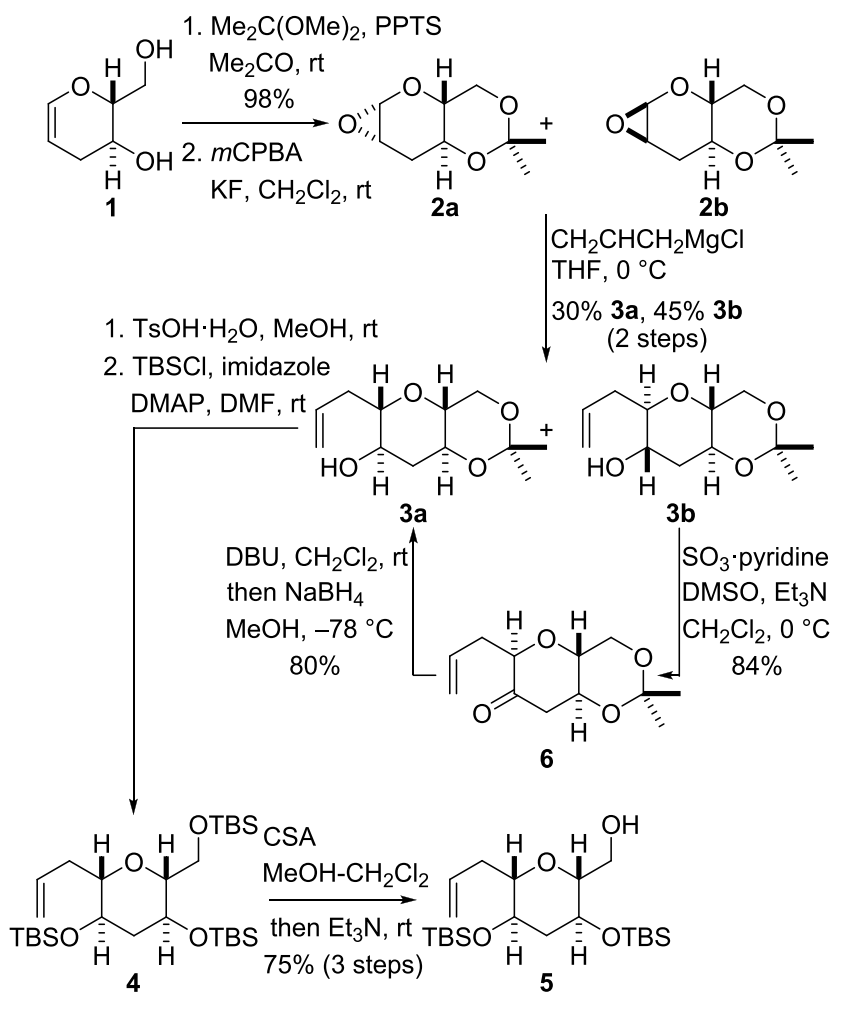

Scheme 2. Preparation of the Bidirectional RCM Precursors $11 a-c$ and 12 from the Alcohol 5

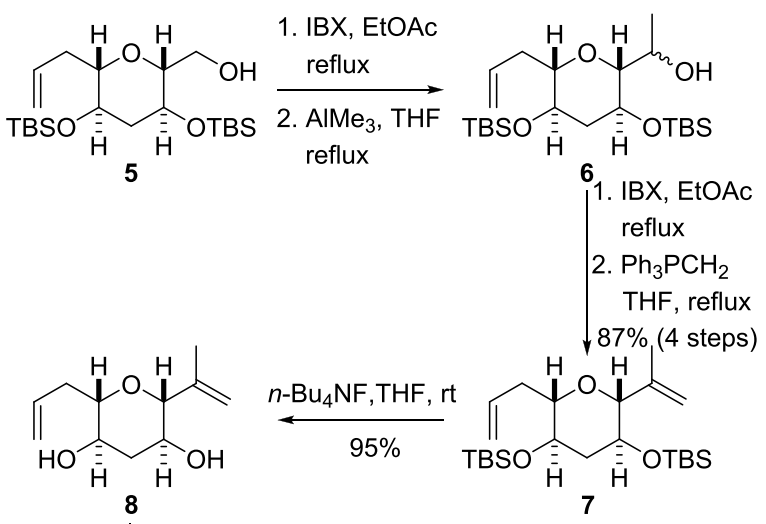

$\mathrm{NaH}, \mathrm{THF}, \mathrm{rt}$

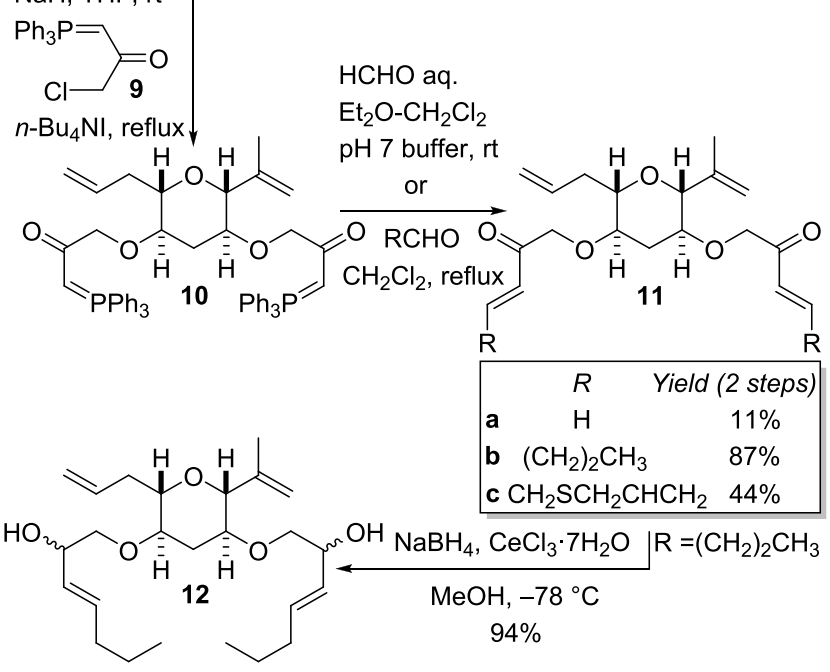


alcohol 6 by oxidation and addition of trimethylaluminum to the resulting aldehyde. ${ }^{15}$ Oxidation of the alcohol to give the corresponding ketone was followed by Wittig methylenation to produce the diene 7 . Cleavage of both silyl ethers was accomplished by treatment of the diene 7 with tetra- $n$ butylammonium fluoride. The resulting diol $\mathbf{8}$ was then subjected to double alkylation with $\alpha$-chloroketophosphorane 9. ${ }^{11,16}$ The resulting bis-ylide $\mathbf{1 0}$ was then reacted with formaldehyde, butanal, or 2-(2-propen-1-ylthio)acetaldehyde ${ }^{17}$ to give the RCM substrates $11 \mathbf{a}-\mathbf{c}$ with variable yields. Subsequent reduction of the bis-enone $\mathbf{1 1 b}$ under Luche conditions delivered the diol 12, the fourth RCM substrate required for exploration of the bidirectional strategy, as a complex mixture comprising all four diastereomers.

The proposed bidirectional RCM reaction to form rings I and $\mathrm{K}$ simultaneously was challenging because it involves the formation of two medium-sized rings, one of the reacting alkenes is 1,1-disubstituted, and there are potential reactivity issues when enones are used as substrates in RCM reactions. ${ }^{18}$ Bidirectional RCM reactions of all four substrates $(11 \mathrm{a}-\mathrm{c}$ and 12) were explored; the Hoveyda-Grubbs second generation complex (13) was used because we had found it to be the optimum catalyst for the cyclization of simpler substrates to produce medium-sized cyclic ethers (Scheme 3). ${ }^{11}$ The RCM

Scheme 3. Bidirectional RCM Reactions of the Substrates $11 \mathrm{a}$ and 12

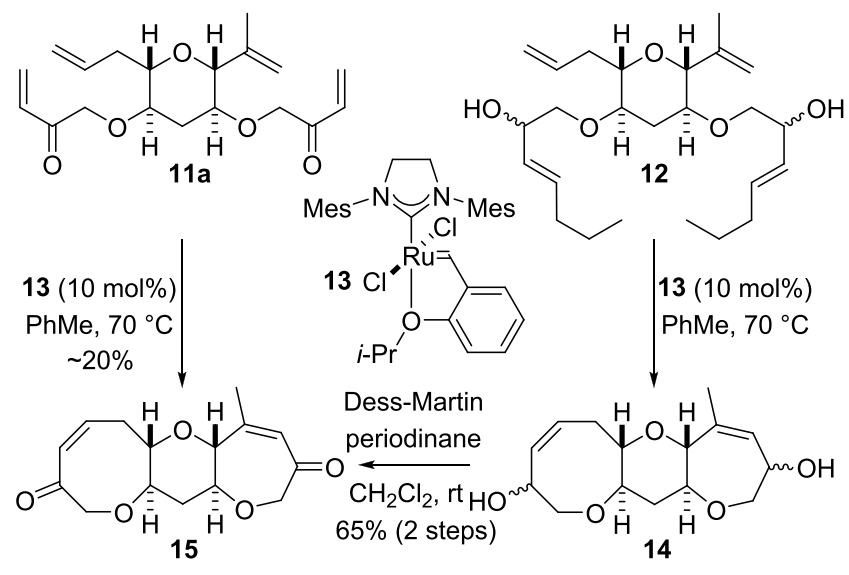

reaction of substrate 11a was low-yielding (yield $\sim 20 \%$ ) as was the reaction used to prepare the substrate, and so the sequence was not viable. Cyclization of substrate $\mathbf{1 1} \mathbf{b}$ by double RCM was performed with the expectation that the propyl groups would direct intermolecular formation of the intermediate ruthenium carbenes to the terminal alkenes instead of the enones, ${ }^{18,19}$ thereby avoiding the generation of potentially less reactive enone-derived alkylidenes. Unfortunately, reaction of the substrate $\mathbf{1 1 b}$ with the complex 13 did not produce the required tricyclic product 15 . The possibility of performing relay RCM in order to favor alkylidene formation at the enone sites was also explored. The substrate 11c, which incorporates an allylic sulfide, was chosen because allylic sulfides are known to be particularly reactive substrates in RCM reactions mediated by the Hoveyda-Grubbs second generation complex (13). ${ }^{20}$ However, attempts to perform bidirectional relay RCM of the substrate 11c were thwarted by degradation of this substrate instead of formation of the required tricyclic product 15. ${ }^{21}$ Finally, we explored the cyclization of the diol 12 with the expectation that the allylic alcohols would be more reactive
RCM partners than the corresponding enones (Scheme 3). ${ }^{11}$ This approach was highly successful, and the diastereomeric mixture of the tetraene 12 underwent RCM to give a diastereomeric mixture of the tricyclic diol $14 .^{22}$ Immediate oxidation of the diastereomeric mixture of diols produced the bis-enone $\mathbf{1 5}$ in $65 \%$ yield over two steps. The RCM reaction was sensitive to temperature, concentration, and catalyst loading. ${ }^{23}$ Reduction of either the reaction temperature or catalyst loading resulted in a lower yield of the diol 14 and the isolation of significant amounts of the bicyclic product in which the eight-membered ring had formed, but the sevenmembered ring had not.

The final sequence required to complete the IJK fragment was conversion of each carbonyl group of the bis-enone $\mathbf{1 5}$ into an enol carbonate followed by a double Tsuji-Trost reaction to install the allyl side chains simultaneously (Scheme 4$).{ }^{10,11}$

Scheme 4. Bidirectional Functionalization of the IJK Ring System

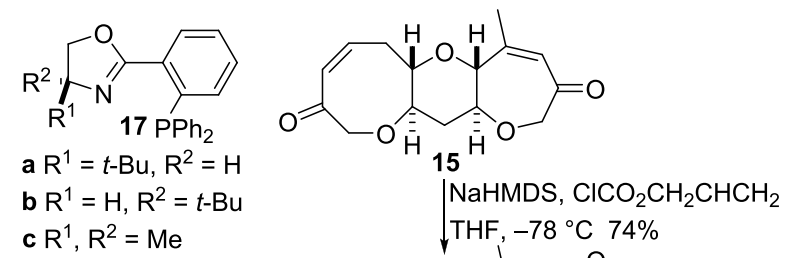

$R^{1}, R^{2}=M e$

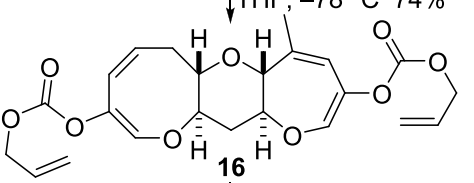

$\left(\mathrm{Ph}_{3} \mathrm{P}\right)_{4} \mathrm{Pd}(20 \mathrm{~mol} \%)$

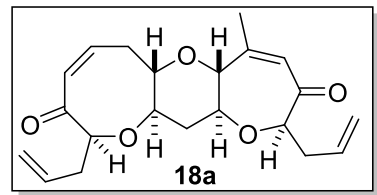

$17(50 \mathrm{~mol} \%), \mathrm{THF}, \mathrm{rt}$
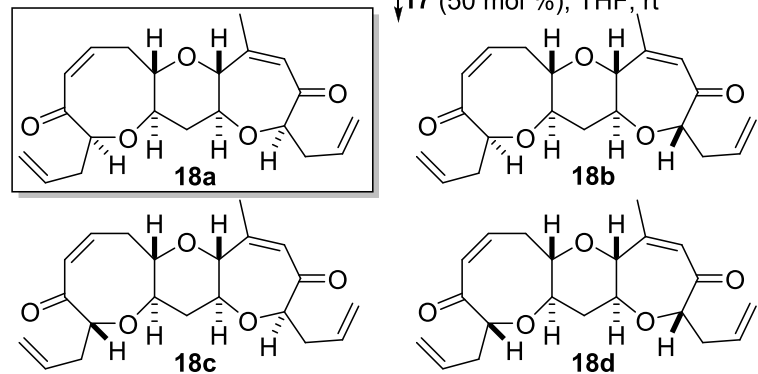

The tricyclic bis-enone $\mathbf{1 5}$ was converted into the precursor (16) required for the allylation reaction by treatment with sodium bis(trimethylsilyl)amide and in situ trapping of the resulting enolates with allyl chloroformate at low temperature. ${ }^{24}$ The resulting bis-carbonate 16 was then subjected to Tsuji-Trost allylation reactions mediated by palladium complexes of the PHOX ligands 17a-c (Scheme 4, Table 1). ${ }^{11}$ The reaction mediated by the achiral dimethyl-PHOX ligand $17 \mathrm{c}$ delivered a mixture of the diastereomers $18 \mathrm{a}-\mathrm{c}$ (entry 1, Table 1). ${ }^{25}$ The product ratio suggests that the level of substrate stereocontrol for allylation in the eight-membered ring is just 54:46 (favoring $S$ configuration at the newly created stereogenic center), an outcome that is consistent with the low level of stereocontrol obtained when a similar fused eightmembered cyclic ether was allylated under these reaction conditions. In the case of the seven-membered ring, data indicate that substrate stereocontrol is approximately 91:9 (favoring $R$ configuration at the newly created stereogenic center), which is significantly higher than the level of stereocontrol obtained when the allylation reaction was 
Table 1. Double Tsuji-Trost Allylation Reactions of bisCarbonate 16 Catalyzed by Palladium Complexes of Ligands $17 \mathrm{a}-\mathrm{c}$

\begin{tabular}{clccccc} 
& & \multicolumn{5}{c}{ Product ratio $^{a}$} \\
\cline { 3 - 6 } Entry & Ligand & $\mathbf{1 8 a}$ & $\mathbf{1 8 b}$ & $\mathbf{1 8 c}$ & $\mathbf{1 8 d}$ & Yield $^{b}$ \\
1 & $\mathbf{1 7 c}$ & 45 & 9 & 46 & - & - \\
2 & $\mathbf{1 7 a}$ & 40 & 49 & 11 & - & $75 \%$ \\
3 & $\mathbf{1 7 b}$ & 37 & - & 63 & - & $70 \%$ \\
4 & $( \pm)-17 \mathbf{a} / \mathbf{b}$ & 42 & 7 & 51 & - & -
\end{tabular}

${ }^{a}$ Ratio of diastereomeric products $18 \mathbf{1}-\mathbf{d}$ determined by ${ }^{1} \mathrm{H}$ NMR analysis of crude material. ${ }^{b}$ Combined yield of diastereomeric products 18 isolated by chromatography.

performed on an analogous but simpler fused seven-membered cyclic ether that lacked the methyl substituent on the enone. ${ }^{11}$

Now that the degree of substrate control had been established by use of an achiral catalyst to promote allylation, Tsuji-Trost reactions were performed with palladium catalysts prepared from the enantiomerically pure chiral PHOX ligands $17 \mathbf{a}$ and $\mathbf{b}$ in order to discover whether it is possible to bias the reaction in favor of the required diastereomer 18a. The relative configuration of the stereogenic centers in the chiral tricyclic substrate $\mathbf{1 6}$ meant that in each case the catalyst and substrate chirality were matched at one site of allylation and mismatched at the other. However, at the outset it was not clear if the stereochemical outcome of the allylation reaction at one site would influence the level of stereocontrol at the other site. In the case of the reaction mediated by the complex of $(S)$-ligand 17a, an excellent level of diastereocontrol (89:11; $\{1 \mathbf{1 8 a}+$ $18 \mathbf{b}\}: 18 c$ ) was obtained for allylation of the eight-membered cyclic ether as a consequence of the matching of the catalyst and substrate (entry 2, Table 1). In the case of the sevenmembered ring, a 51:49 ratio of isomers was obtained favoring the required $R$ configuration at the newly formed stereogenic center. Thus, the mismatch between the catalyst and substrate had completely eroded substrate control (originally 90:10 in favor of the $R$ configuration) in the seven-membered ring. The situation was reversed when the reaction was performed with the palladium complex of the $(R$ )-ligand $\mathbf{1 7 b}$ (entry 3, Table $1)$. Complete control of the configuration of the stereogenic center on the seven-membered ring was achieved, and the diastereomers 18a and $18 \mathrm{c}$ (37:63 ratio) were produced, which differed in their configuration at the stereogenic center in the eight-membered ring. In this case, the level of intrinsic diastereocontrol in the eight-membered ring was lower and so the catalyst was able to over-ride this weak substrate bias to a significant extent.

To complete catalyst screening, we also performed an allylation reaction mediated by the palladium complex generated from a racemic mixture of ligands $17 \mathbf{a} / \mathbf{b}$ (entry 4 , Table 1). The tricyclic substrate $\mathbf{1 6}$ is chiral, and it is conceivable that the stereochemical outcome of the reaction might be different when a racemic catalyst is used instead of an achiral catalyst, particularly if the carbonate groups in the chiral substrate 16 react with the enantiomers of the racemic catalyst at different rates. The product distribution obtained from the reaction mediated by the racemic catalyst was similar but not identical to that produced when the reaction was performed using the achiral catalyst (entry 1, Table 1). However, differences in the product distribution obtained from the two reactions (entries 1 and 4, Table 1) are relatively small and could arise either from structural differences between the achiral and racemic catalysts or measuring errors when determining the ratios of products by ${ }^{1} \mathrm{H}$ NMR analysis, so it is unclear whether the differences are significant or not.

In summary, we have shown that the IJK fragment 18a of the polyether natural product CTX3C can be prepared from a simple monocyclic precursor 12 in a concise and efficient manner by use of a four-step bidirectional sequence that involves RCM, alcohol oxidation, enol carbonate formation, and palladium-mediated Tsuji-Trost allylation. The double allylation reaction can be directed to give either $\mathbf{1 8 a}$ and $\mathbf{1 8 b}$ or 18a and 18c, by selection of the appropriate PHOX ligand $(17 \mathbf{a}$ or $17 \mathbf{b})$, but it is not possible to obtain the diastereomer 18a with high selectivity. This finding suggests that in order to obtain the required diastereomer 18a selectively, it will be necessary to conduct the Tsuji-Trost allylation reactions sequentially instead of simultaneously, or perform epimerization of the mixture of diastereomers produced by the double allylation reaction. ${ }^{26}$

\section{ASSOCIATED CONTENT}

\section{Supporting Information}

The Supporting Information is available free of charge at https://pubs.acs.org/doi/10.1021/acs.orglett.0c01238.

Full experimental details for the preparation of all new compounds and spectroscopic data (PDF)

\section{AUTHOR INFORMATION}

\section{Corresponding Author}

J. Stephen Clark - School of Chemistry, Joseph Black Building, University of Glasgow, Glasgow G12 8QQ United Kingdom; ○ orcid.org/0000-0003-3935-0377; Email: stephen.clark@ glasgow.ac.uk

\section{Authors}

Michael Popadynec - School of Chemistry, Joseph Black Building, University of Glasgow, Glasgow G12 8QQ, United Kingdom

Helen Gibbard - School of Chemistry, Joseph Black Building, University of Glasgow, Glasgow G12 8QQ, United Kingdom

Complete contact information is available at:

https://pubs.acs.org/10.1021/acs.orglett.0c01238

Notes

The authors declare no competing financial interest.

\section{ACKNOWLEDGMENTS}

The authors gratefully acknowledge studentship funding from EPSRC (Grant Numbers EP/P50418X, EP/P504937/1, EP/ K503058, and EP/L50497X) to support M.P. and H.G.

\section{REFERENCES}

(1) Satake, M.; Murata, M.; Yasumoto, T. The Structure of CTX3C, a Ciguatoxin Congener Isolated from Cultured Gambierdiscus Toxicus. Tetrahedron Lett. 1993, 34, 1975-1978.

(2) Caillaud, A.; de la Iglesia, P.; Darius, H. T.; Pauillac, S.; Aligizaki, K.; Fraga, S.; Chinain, M.; Diogène, J. Update on Methodologies Available for Ciguatoxin Determination: Perspectives to Confront the Onset of Ciguatera Fish Poisoning in Europe. Mar. Drugs 2010, 8, 1838-1907.

(3) Ghiaroni, V.; Fuwa, H.; Inoue, M.; Sasaki, M.; Miyazaki, K.; Hirama, M.; Yasumoto, T.; Rossini, G. P.; Scalera, G.; Bigiani, A. 
Effect of Ciguatoxin 3C on Voltage-Gated $\mathrm{Na}^{+}$and $\mathrm{K}^{+}$Currents in Mouse Taste Cells. Chem. Senses 2006, 31, 673-680.

(4) Hamilton, B.; Whittle, N.; Shaw, G.; Eaglesham, G.; Moore, M. R.; Lewis, R. J. Human Fatality Associated with Pacific Ciguatoxin Contaminated Fish. Toxicon 2010, 56, 668-673.

(5) (a) Fujiwara, K.; Goto, A.; Sato, D.; Ohtaniuchi, Y.; Tanaka, H.; Murai, A.; Kawai, H.; Suzuki, T. Convergent Synthesis of the ABCDE-Ring Part of Ciguatoxin CTX3C. Tetrahedron Lett. 2004, 45, 7011-7014. (b) Domon, D.; Fujiwara, K.; Ohtaniuchi, Y.; Takezawa, A.; Takeda, S.; Kawasaki, H.; Murai, A.; Kawai, H.; Suzuki, T. Synthesis of the C42-C52 Part of Ciguatoxin CTX3C. Tetrahedron Lett. 2005, 46, 8279-8283. (c) Domon, D.; Fujiwara, K.; Murai, A.; Kawai, H.; Suzuki, T. Convergent Synthesis of the IJKLM-Ring Part of Ciguatoxin CTX3C. Tetrahedron Lett. 2005, 46, 8285-8288. (d) Takizawa, A.; Fujiwara, K.; Doi, E.; Murai, A.; Kawai, H.; Suzuki, T. Synthesis of the Common FGHI-Ring Part of Ciguatoxins. Tetrahedron Lett. 2006, 47, 747-751. (e) Kadota, I.; Abe, T.; Uni, M.; Takamura, H.; Yamamoto, Y. Convergent Synthesis of the A-E Ring Segment of Ciguatoxin CTX3C. Tetrahedron 2009, 65, 7784-7789. (f) Takamura, H.; Nishiuma, N.; Abe, T.; Kadota, I. Convergent Synthesis of the HIJKLM Ring System of Ciguatoxin CTX3C. Org. Lett. 2011, 13, 4704-4707. (g) Takamura, H.; Abe, T.; Nishiuma, N.; Fujiwara, R.; Tsukeshiba, T.; Kadota, I. A Convergent Synthesis of the Right-Hand Fragment of Ciguatoxin CTX3C. Tetrahedron 2012, 68, 2245-2260. (h) Shiroma, K.; Asakura, H.; Tanaka, T.; Takamura, H.; Kadota, I. Improved Synthesis of the A-E Ring Segment of Ciguatoxin CTX3C. Heterocycles 2014, 88, 969-973. (i) Kadota, I.; Sato, Y.; Fujita, N.; Takamura, H.; Yamamoto, Y. Convergent Synthesis of the EFGH Ring System of Ciguatoxin CTX3C. Tetrahedron 2015, 71, 6547-6558. (j) Tanaka, T.; Asakura, H.; Fujiwara, R.; Kumamoto, K.; Izuka, H.; Shiroma, K.; Takamura, H.; Kadota, I. Improved Synthesis of the A-E Ring Segment of Ciguatoxin CTX3C by Using Intramolecular Allylations. Bull. Chem. Soc. Jpn. 2018, 91, 507-514. (k) Saito, T.; Fujiwara, K.; Sano, Y.; Sato, T.; Kondo, Y.; Akiba, U.; Ishigaki, Y.; Katoono, R.; Suzuki, T. An Improved Synthesis of the C42-C52 Segment of Ciguatoxin 3C. Tetrahedron Lett. 2018, 59, 1372-1376.

(6) (a) Hirama, M.; Oishi, T.; Uehara, H.; Inoue, M.; Maruyama, M.; Oguri, H.; Satake, M. Total Synthesis of Ciguatoxin CTX3C. Science 2001, 294, 1904-1907. (b) Inoue, M.; Uehara, H.; Maruyama, M.; Hirama, M. Practical Total Synthesis of Ciguatoxin CTX3C by Improved Protective Group Strategy. Org. Lett. 2002, 4, 4551-4554. (c) Inoue, M.; Miyazaki, K.; Uehara, H.; Maruyama, M.; Hirama, M. First- and Second-Generation Total Synthesis of Ciguatoxin CTX3C. Proc. Natl. Acad. Sci. U. S. A. 2004, 101, 12013-12018. (d) Inoue, M.; Hirama, M. Evolution of a Practical Total Synthesis of Ciguatoxin CTX3C. Acc. Chem. Res. 2004, 37, 961-968. (e) Inoue, M.; Miyazaki, K.; Ishihara, Y.; Tatami, A.; Ohnuma, Y.; Kawada, Y.; Komano, K.; Yamashita, S.; Lee, N.; Hirama, M. Total Synthesis of Ciguatoxin and 51-HydroxyCTX3C. J. Am. Chem. Soc. 2006, 128, 9352-9354.

(7) Hamajima, A.; Isobe, M. Total Synthesis of Ciguatoxin. Angew. Chem., Int. Ed. 2009, 48, 2941-2945.

(8) (a) Clark, J. S.; Kimber, M. C.; Robertson, J.; McErlean, C. S. P.; Wilson, C. Rapid Two-Directional Synthesis of the F-J Fragment of the Gambieric Acids by Iterative Double Ring-Closing Metathesis. Angew. Chem., Int. Ed. 2005, 44, 6157-6162. (b) Clark, J. S.; Romiti, F.; Sieng, B.; Paterson, L. C.; Stewart, A.; Chaudhury, S.; Thomas, L. H. Synthesis of the A-D Ring System of Gambieric Acids. Org. Lett. 2015, 17, 4694-4697.

(9) Clark, J. S.; Conroy, J.; Blake, A. J. Rapid Synthesis of the A-E Fragment of Ciguatoxin CTX3C. Org. Lett. 2007, 9, 2091-2094.

(10) (a) Behenna, D. C.; Stoltz, B. M. The Enantioselective Tsuji Allylation. J. Am. Chem. Soc. 2004, 126, 15044-15045. (b) McFadden, R. M.; Stoltz, B. M. The Catalytic Enantioselective, Protecting GroupFree Total Synthesis of (+)-Dichroanone. J. Am. Chem. Soc. 2006, 128, 7738-7739. (c) Mohr, J. T.; Stoltz, B. M. Enantioselective Tsuji Allylations. Chem. Asian J. 2007, 2, 1476-1491. (d) Enquist, J. A.; Stoltz, B. M. The Total Synthesis of (-)-Cyanthiwigin F by Means of a Double Catalytic Enantioselective Alkylation. Nature 2008, 453, $1228-1231$.

(11) Skardon-Duncan, J.; Sparenberg, M.; Bayle, A.; Alexander, S.; Clark, J. S. Stereoselective Synthesis of Medium-Sized Cyclic Ethers by Sequential Ring-Closing Metathesis and Tsuji-Trost Allylation. Org. Lett. 2018, 20, 2782-2786.

(12) Bellucci, G.; Catelani, G.; Chiappe, C.; D’Andrea, F. A Simple and Highly Diastereoselctive Preparation of Glycal Epoxides Using the $m$ CPBA-KF Complex. Tetrahedron Lett. 1994, 35, 8433-8436.

(13) Halcomb, R. L.; Danishefsky, S. J. On the Direct Epoxidation of Glycals: Application of a Reiterative Strategy for the Synthesis of $\beta$ Linked Oligosaccharides. J. Am. Chem. Soc. 1989, 111, 6661-6666.

(14) Sasaki, M.; Koike, T.; Sakai, R.; Tachibana, K. Total Synthesis of (-)-Dysiherbaine, a Novel Neuroexcitotoxic Amino Acid. Tetrahedron Lett. 2000, 41, 3923-3926.

(15) Reaction of the aldehyde 5 with methylmagnesium bromide resulted in an inferior $(60 \%)$ yield of the alcohol 6.

(16) (a) Cossy, J.; Taillier, C.; Bellosta, V. Synthesis of 3-Oxo Oxacycloalkenes by Ring Closing Metathesis. Tetrahedron Lett. 2002, 43, 7263-7266. (b) Taillier, C.; Hameury, T.; Bellosta, V.; Cossy, J. Synthesis of 3-Oxooxa- and 3-Oxoazacycloalk-4-enes by Ring Closing Metathesis. Application to the Synthesis of an Inhibitor of Cathepsin K. Tetrahedron 2007, 63, 4472-4490.

(17) Aurich, H. G.; Möbus, K.-D. Diastereoselective Synthesis of $\alpha, \gamma$-Amino Alcohols by Intramolecular 1,3-Dipolar Cycloaddition of Nitrones Containing an Allylthio Group Followed by Reductive RingOpening. Tetrahedron Lett. 1988, 29, 5755-5758.

(18) Boiteau, J.-G.; Van de Weghe, P.; Eustache, J. A New, Ring Closing Metathesis-Based Synthesis of (-)-Fumagillol. Org. Lett. 2001, 3, 2737-2740.

(19) The propyl substituents in $\mathbf{1 1} \mathbf{b}$ were selected because reaction of the bis-ylide $\mathbf{1 0}$ with a relatively nonvolatile simple aldehyde (butanal) delivered the highest yields of 11 .

(20) (a) Edwards, G. A.; Culp, P. A.; Chalker, J. M. Allyl Sulphides in Olefin Metathesis: Catalyst Considerations and Traceless Promotion of Ring-Closing Metathesis. Chem. Commun. 2015, 51, 515-518. (b) Chalker, J. M. Allyl Sulfides: Reactive Substrates for Olefin Metathesis. Aust. J. Chem. 2015, 68, 1801-1809.

(21) A complex mixture of unidentifiable products was obtained from the RCM reaction.

(22) The diol 12 was obtained as a mixture of the four possible diastereomers in approximately equivalent amounts. The good yield obtained for the RCM and oxidation sequence suggests that all four diastereomers underwent RCM, but the precise ratio of isomers was not determined prior to the oxidation reaction.

(23) RCM reactions of the diol $\mathbf{1 2}$ were performed in toluene at a concentration of $1 \mathrm{mM}$.

(24) Formation of the $\mathrm{O}$-acylation product arising from competitive $\gamma$-deprotonation of the methyl substituent was not observed.

(25) The diastereomer 18d, which is expected to be the least kinetically accessible and the least thermodynamically stable of the four possible diastereomers of $\mathbf{1 8}$, was not isolated from any of the allylation reactions.

(26) Treatment of the close analogue of diketone $\mathbf{1 8}$ that lacks the K-ring methyl substituent with DBU in toluene produced a mixture of two diastereomers (6:1 ratio) favoring the isomer with the required configuration. 